\title{
Kinesin light chain 4 as a new target for lung cancer chemoresistance via targeted inhibition of checkpoint kinases in the DNA repair network
}

\author{
Jeong-Hwa Baek', Hong Shik Yun², Ju-Young Kim³,4, Janet Lee ${ }^{3}$, Yeon-Joo Lee $\mathbb{B}^{3}$, Chang-Woo Lee ${ }^{4}$,

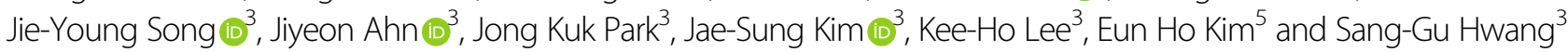

\begin{abstract}
The poor therapeutic efficacy of non-small cell lung cancer (NSCLC) is partly attributed to the acquisition of chemoresistance. To investigate the mechanism underlying this resistance, we examined the potential link between kinesin light chain 4 (KLC4), which we have previously reported to be associated with radioresistance in NSCLC, and sensitivity to chemotherapy in human lung cancer cell lines. KLC4 protein levels in lung cancer cells correlated with the degree of chemoresistance to cisplatin treatment. Furthermore, KLC4 silencing enhanced the cytotoxic effect of cisplatin by promoting DNA double-strand breaks and apoptosis. These effects were mediated by interaction with the checkpoint kinase CHK2, as KLC4 knockdown increased CHK2 activation, which was further enhanced in combination with cisplatin treatment. In addition, KLC4 and CHEK2 expression levels showed negative correlation in lung tumor samples from patients, and $K L C 4$ overexpression correlated negatively with survival. Our results indicate a novel link between the KLC4 and CHK2 pathways regulating DNA damage response in chemoresistance, and highlight KLC4 as a candidate for developing lung cancer-specific drugs and customized targeted molecular therapy.
\end{abstract}

\section{Introduction}

Lung cancer is one of the main reasons of cancerrelated deaths worldwide, and $80 \%$ cases of lung cancer are non-small cell lung cancer (NSCLC) with poor prognosis at diagnosis and limited therapeutic efficiency $^{1,2}$. Current research directions for lung cancer treatment are inclusive of immunotherapy, which makes it possible for the body's immune system to attack the tumor cells, epigenetics, and new combinations of chemotherapy and radiotherapy, both on their own and collectively. Many of these new treatments work through immune checkpoint blockade, thus disrupting cancer's

\footnotetext{
Correspondence: Eun Ho Kim (eh140149@cu.ac.kr) or

Sang-Gu Hwang (sgh63@kcch.re.kr)

${ }^{1}$ Radiation Biology Research Team, Research Center, Dongnam Institute of

Radiological and Medical Sciences, Busan 46033, Republic of Korea

${ }^{2}$ Radiation Oncology Branch, National Cancer Institute, NIH, Bethesda, MD, USA

Full list of author information is available at the end of the article

These authors contributed equally: Eun Ho Kim, Sang-Gu Hwang

Edited by M. Malewicz
}

ability to evade the immune system ${ }^{3,4}$. Targeted therapy and immunotherapy are known to significantly prolong the 5-year survival, and more than $17 \%$ patients can survive for 5 years after being diagnosed with advanced stage NSCLC ${ }^{5}$. However, chemoresistance for lung cancer treatment remains an important barrier to treatment efficacy $^{6,7}$. Lung cancer is well-known to have high recurrence rate, as lung cancer cells cannot be entirely removed using conventional chemotherapeutics owing to the development of drug resistance ${ }^{8}$. Cisplatin can cause interstrand and intrastrand crosslinks between purine bases; therefore, it is widely used for treating solid tumors9. However, chronic treatment with cisplatin can induce a chemoresistant phenotype. Therefore, identifying the genes underlying this chemoresistant phenotype is critical for better understanding of the molecular pathogenesis of NSCLC and for developing gene-targeted therapies aimed at treating chemoresistant cancer. Furthermore, elucidating the molecular mechanisms of

\section{(c) The Author(s) 2020}

(c) (i) Open Access This article is licensed under a Creative Commons Attribution 4.0 International License, which permits use, sharing, adaptation, distribution and reproduction cc) in any medium or format, as long as you give appropriate credit to the original author(s) and the source, provide a link to the Creative Commons license, and indicate if changes were made. The images or other third party material in this article are included in the article's Creative Commons license, unless indicated otherwise in a credit line to the material. If material is not included in the article's Creative Commons license and your intended use is not permitted by statutory regulation or exceeds the permitted use, you will need to obtain permission directly from the copyright holder. To view a copy of this license, visit http://creativecommons.org/licenses/by/4.0/. 
cisplatin resistance will assist in establishing effective prognostic biomarkers and enhancing the efficiency of related therapeutic interventions.

Toward this, extensive investigations have focused on uncovering the molecular mechanism contributing to the initiation and progression of NSCLC, resulting in the development of diverse novel targeted agents such as epidermal growth factor receptor tyrosine kinase inhibitors, which are more efficient than chemotherapy in patients with epidermal growth factor receptor-mutated tumors ${ }^{10}$. Despite numerous experimental studies in this field, studies focusing on the mechanisms of resistance based on clinical therapy are scarce and generally include only a small number of patients.

Checkpoint kinases (CHK1 and CHK2) have recently emerged as potential new targets for cancer therapy to enhance response to genotoxic drugs. CHK1 and CHK2 play critical roles in the DNA damage response (DDR) network $^{11}$, and DNA repair pathways are important for resistance to DNA-damaging cytotoxic therapy and radiation $^{12}$. Cellular defenses against DNA damage are regulated by multiple checkpoints that enable cell cycle arrest, DNA repair, or apoptosis in case of extensive DNA damage ${ }^{13,14}$. CHK2 plays particularly vital roles in DDR via signaling of the ATM-CHK2-P53 pathway, and in regulating cell cycle checkpoints, including the G2/M checkpoint ${ }^{15,16}$. CHK2 phosphorylates multiple target proteins that are involved in oncogenesis, including p53, CDC25A, CDC25C, BRCA1, E2F1, and MDC1 ${ }^{11}$. Support for the role of $\mathrm{CHK} 2$ in cancer comes from rare germline or somatic mutations in $C H K 2$ in certain human familial cancers and several tumor types, and from its important role in oncogene-induced senescence ${ }^{16}$. Furthermore, several reports indicate the advantage of CHK2 inhibition in inducing tumor killing in response to genotoxic drugs ${ }^{15}$. CHK2 has been verified as a tumor suppressor, and is mutated or depleted in several cancers, including breast, colon, bladder, ovarian, and prostate carcinomas ${ }^{17,18}$. In addition, low level of CHK2 in lung cancers was suggested to contribute to chemo-radiation resistance ${ }^{19}$.
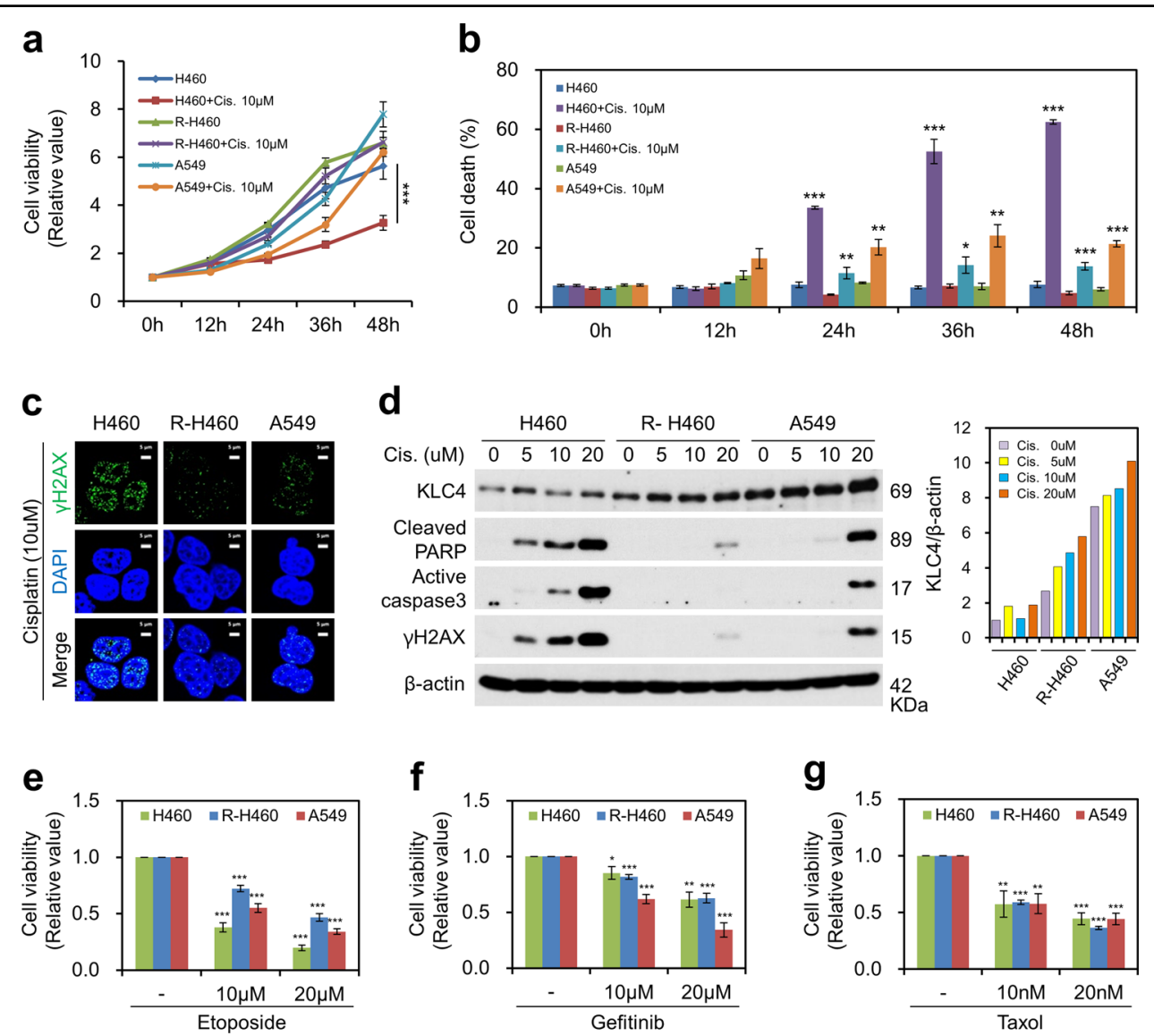

Fig. 1 KLC4 was related to chemoresistance in vitro. a Cell viability was measured every $12 \mathrm{~h}$ after treatment with $10 \mu \mathrm{M}$ cisplatin. $\mathbf{b}$ Death of cells treated with or without $10 \mu \mathrm{M}$ cisplatin during $12 \mathrm{~h}$ intervals. c After cisplatin treatment for $24 \mathrm{~h}$, the cells were fixed with $4 \%$ paraformaldehyde and immunostained using an antibody targeting YH2AX (DNA damage marker); DNA was visualized using DAPI staining. $\mathbf{d}$ Protein levels of KLC4, cleaved PARP, and active caspase-3 were determined using western blotting after cisplatin treatment. e- $\mathbf{g}$ Cells treated with etoposide, gefitinib, or Taxol, respectively. Twenty-four hours after the drug treatments, the cells were analyzed using cell viability assay, followed by ELISA. 
Recently, we identified several proteins, including kinesin light chain 4 (KLC4), to be involved in the radioresistance of $\mathrm{NSCLC}^{20}$. However, the regulatory mechanism linking KLC4 expression and sensitivity to chemotherapy or radioresistance in lung cancer remains unclear. We first investigated whether KLC4 expression and sensitivity to chemotherapy or radioresistance in lung cancer cell lines treated with cisplatin or other common chemotherapy drugs were related. We further hypothesized that KLC4 may be involved in the DDR via interaction with CHK1/2 to drive chemoresistance. Therefore, we investigated the effect of KLC4 knockdown on CHK1/ 2 activation, cytotoxicity, and DNA damage induction by cisplatin. Our study highlights a new candidate for the development of lung cancer-specific drugs and customized targeted molecular therapy.

\section{Results}

KLC4 regulated chemoresistance in lung cancer cells

We first evaluated the anticancer drug resistance of the lung cancer cell lines, $\mathrm{H} 460$ with lower KLC4 expression, and R-H460 and A549 with higher KLC4 expression than that of $\mathrm{H} 460$ cells. We assessed the effect of cisplatin treatment on cell growth and proliferation of the three lung cancer cell lines. The cell viability assay showed that $10 \mu \mathrm{M}$ cisplatin (treated for $0,12,24,36$, and $48 \mathrm{~h}$ ) significantly $(P<0.001)$ inhibited the growth of $\mathrm{H} 460$ cells (Fig. 1a).
As shown in Fig. 1b, cisplatin-induced considerable apoptosis in $\mathrm{H} 460$ cells; however, compared with that in $\mathrm{H} 460$ cells, apoptosis was not significantly increased in R-H460 and A549 cells. To investigate whether cisplatin as a DNA damage inducer altered double strand break (DSB) repair, we attempted to validate DDR according to KLC4 expression in the lung cancer cell lines. We monitored the formation of $\gamma-\mathrm{H} 2 \mathrm{AX}$ foci in the cisplatintreated cells and observed an increase in the number of $\gamma$ H2AX foci after 24h (Fig. 1c). Furthermore, compared with those in R-H460 and A549 cells, cisplatin significantly increased the number of $\gamma-\mathrm{H} 2 \mathrm{AX}$ foci in $\mathrm{H} 460$ cells (Fig. 1c). Western blotting further showed that KLC4 protein levels in human lung cancer cell lines (H460, RH460, and A549 cells) correlated with the tendency of chemoresistance (Fig. 1d). H460 cells were more sensitive to cisplatin than R-H460 and A549 cells. These results indicated a correlation between increase in KLC4 expression and chemoresistance. Several lines of evidence suggest that lung cancer cells are resistant to multiple therapeutic agents ${ }^{21}$. Therefore, we further assessed the inhibition of cell viability of a spectrum of chemoresistant lung cancer cells (H460, R-H460, and A549 cells) treated with conventional chemotherapeutic drugs that are wellknown inducers of apoptosis, including etoposide (DNAdamaging agent), gefitinib (EGFR inhibitor), and Taxol (microtubule inhibitor), for $24 \mathrm{~h}^{22}$. Similar to the effect of cisplatin, only etoposide, but not gefitinib or Taxol, a

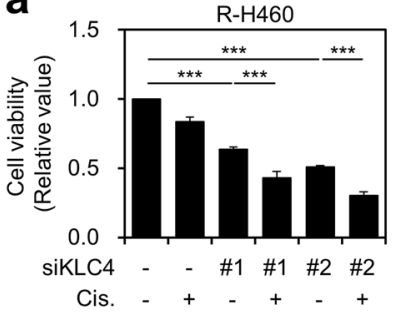

d

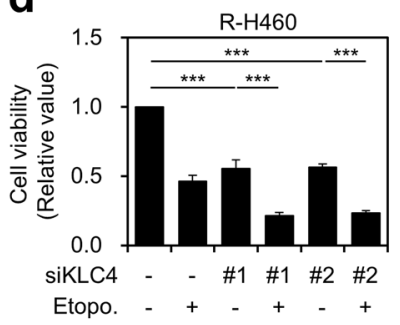

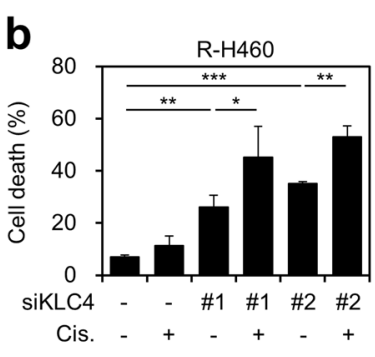

e

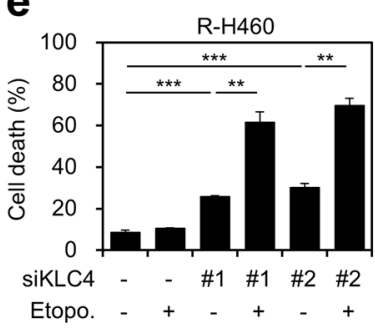

C

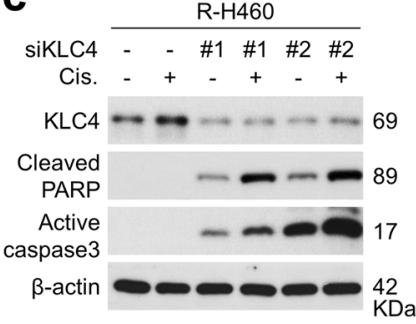

$\mathbf{f}$

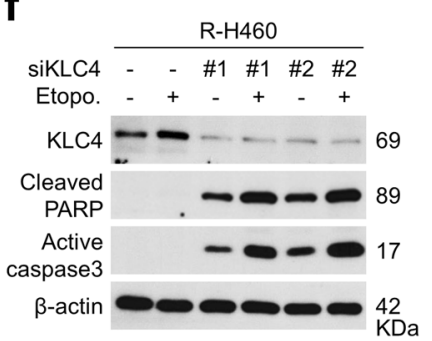

Fig. 2 KLC4 depletion reversed chemoresistance in lung cancer cells. a Viability of R-H460 cells treated with or without $10 \mu \mathrm{M}$ cisplatin after transfection with siCON (negative control) or siKLC4. b Cell death in R-H460 cells [treated as described in (a)] using annexin V/propidium iodide staining. c Protein levels of KLC4, cleaved PARP, and active caspase-3 (cell death marker) as determined using western blotting. $\mathbf{d}$ Viability of R-H460 cells treated with or without $10 \mu \mathrm{M}$ etoposide after transfection with siCON or siKLC4. e-f R-H460 cells were treated with or without $10 \mu \mathrm{M}$ etoposide after transfection with $K L C 4$ siRNA. Cell death was measured $48 \mathrm{~h}$ after treatment using annexin V/propidium iodide staining (e), and western blotting (f) 

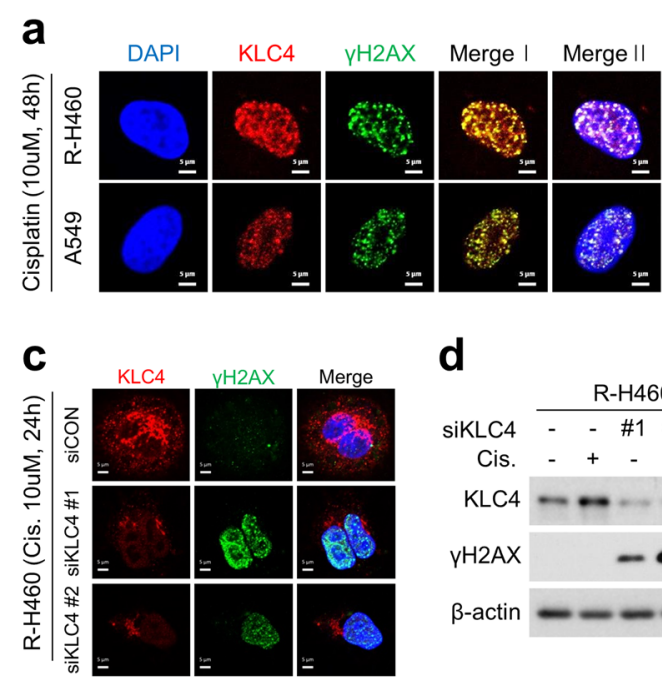

d

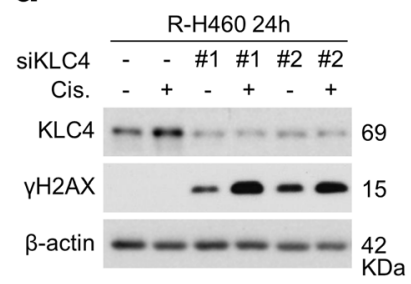

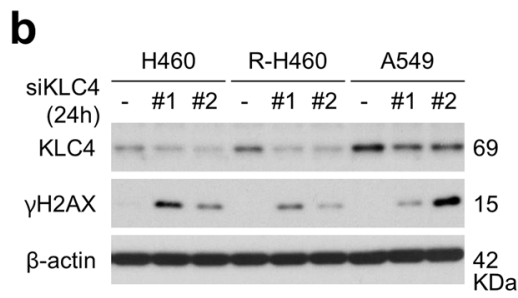

e

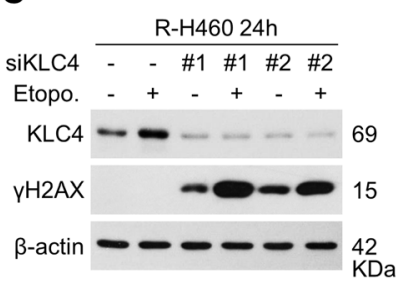

f

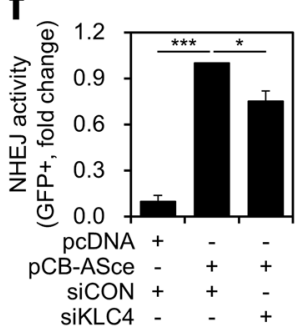

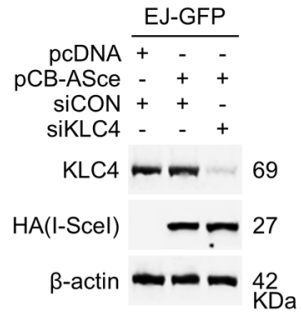

g

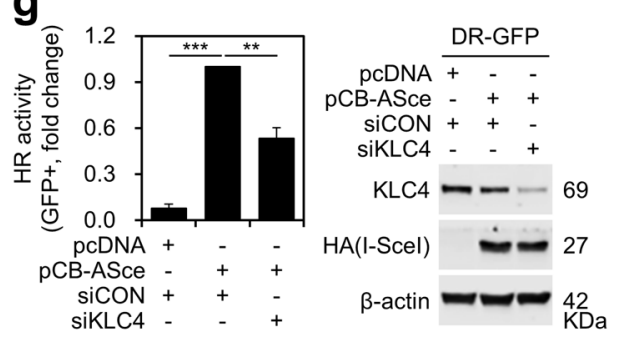

Fig. 3 Downregulation of $K L C 4$ induced DNA damage response. a Cells were fixed with $4 \%$ paraformaldehyde and stained using antibodies targeting KLC4 and $\mathrm{YH} 2 \mathrm{AX}$; DNA was visualized using DAPI staining. b Protein levels of KLC4 and $\mathrm{YH} 2 \mathrm{AX}$ were determined using western blotting after transfection with KLC4 siRNA. c R-H460 cells were treated with $10 \mu \mathrm{M}$ cisplatin after transfection with KLC4 siRNA for $24 \mathrm{~h}$. Cells were fixed and immunostained using antibodies targeting KLC4 and $\mathrm{yH} 2 \mathrm{AX}$. d, e R-H460 cells were pre-treated with $10 \mu \mathrm{M}$ cisplatin (d) or $10 \mu \mathrm{M}$ etoposide (e) and transfected with siKLC4. The cell lysates were prepared and used for immunoblotting with antibodies against KLC4 and $\gamma H 2 A X$. $\mathbf{f}, \mathbf{g}$ Reporter cells were transfected with KLC4 siRNA and pCB-ASce vector (HA tagged I-Scel expression). GFP-positive cells were counted using flow cytometry in the U2OS EJ-GFP cell (f) or the U2OS DR-GFP cell $(\mathbf{g})$.

correlated with the expression level of KLC4 and inhibition of cell viability (Fig. 1e-g). Thus, KLC4 is expected to be involved in the positive resistance to DNA damagerelated anticancer drugs.

KLC4 knockdown induced growth inhibition and apoptosis in cisplatin- or etoposide- treated lung cancer cells

To further investigate the effects of KLC4 in regulating the fate of lung cancer cells treated with anticancer drugs, the gene was silenced via RNA interference using specific siRNA targeting KLC4. As illustrated in Fig. 2c and Supplementary Fig. 1c, KLC4 was successfully knocked down in R-H460 and A549 cells after transfection with the KLC4 siRNA. Furthermore, compared with that observed with $K L C 4$ silencing alone in R-H460 and A549 cells, the combination of KLC4 silencing with cisplatin treatment decreased cell viability (Fig. 2a, Supplementary Fig. 1a). The anchorage-dependent colony forming assay showed that KLC4 siRNA plus cisplatin significantly $(P<0.001)$ decreased the number of colonies formed in A549 cells (Supplementary Fig. 2a). Compared with that observed with KLC4 siRNA treatment alone, knockdown of KLC4 in combination with cisplatin also increased lung cancer cell death, as was evident from the evaluation of apoptosis using flow cytometry (Fig. 2b, Supplementary Fig. 1b). In addition, the levels of cleaved PARP and active caspase-3 were higher in KLC4 siRNA-transfected cells combined with cisplatin treatment than in untreated siRNA-transfected cells (Fig. 2c, Supplementary Fig. 1c). Similarly, compared with that observed with KLC4 siRNA treatment alone in R-H460 and A549 cell lines, the combination of etoposide with KLC4 siRNA treatment significantly inhibited cell viability and cell death (Fig. 2d-f, Supplementary Fig. 2d-f). These results showed that KLC4 knockdown enhanced the cytotoxicity of cisplatin and etoposide, indicating $K L C 4$ as a novel chemoresistance gene in lung cancer. 
Knockdown of KLC4 in lung cancer cells enhanced cisplatin or etoposide-induced DNA damage and regulated nonhomologous end joining (NHEJ) repair

Cisplatin can lead to the intracellular accumulation of DNA DSBs and $\gamma-\mathrm{H} 2 \mathrm{AX}$ protein, which is responsible for cell apoptosis ${ }^{23}$. Furthermore, etoposide also induces DNA damage in cells by interacting with the nuclear enzyme topoisomerase $\mathrm{II}^{24}$. Therefore, we further investigated the effect of cisplatin and/or KLC4 expression on $\gamma$-H2AX level, which is the Ser139 phosphorylated form of H2AX and a recognized marker of DNA damage ${ }^{25}$. The immunofluorescence images clearly illustrated that KLC4 colocalized with the cisplatin-induced foci of $\gamma$ H2AX in R-H460 and A549 cells (Fig. 3a). Inhibition of KLC4 expression in the three types of lung cancer cell lines increased the expression level of $\gamma$-H2AX (Fig. 3b). We detected a significant increase in $\gamma$-H2AX foci in the KLC4 siRNA-transfected R-H460 and A549 cells, which gradually increased to the initial level after additional cisplatin treatment (Fig. 3c, Supplementary Fig. 3a). Consistently, transfection of siKLC4, combined with cisplatin or etoposide, markedly enhanced the accumulation of $\gamma-\mathrm{H} 2 \mathrm{AX}$ at the protein level (Fig. 3d, e,
Supplementary Fig. 3b, c), indicating that KLC4 depletion may considerably increase the genotoxic effect of cisplatin and etoposide in aggravating DNA damage in lung cancer cells. Furthermore, in agreement with previous results, KLC4 depletion maintained DNA damage, and thus enhanced the radiosensitivity of the cells (Supplementary Fig. 3d). Subsequently, we used the reporter systems to study non-homologous end joining (NHEJ) and homologous recombination (HR) activity as the primary DNA DSB repair pathways ${ }^{26}$. Using U2OS EJGFP cells harboring a single chromosomally integrated copy of the pimEJ5-GFP construct ${ }^{27-29}$, the percentage of GFP-positive cells was considerably reduced in cells that were treated with KLC4 siRNA after exogenous expression of HA tagged I-SceI, which, in turn, suggested the suppression of the NHEJ repair pathway after KLC4 knockdown (Fig. 3f). Similar to the result of the EJ reporter assay, a considerable change in the GFP-positive rate was detected after silencing KLC4 in the U2OS cells with stable transfection of the pHRPT-DRGRP plasmid (Fig. 3g). These results suggested that KLC4 primarily affected all of the NHEJ and also the HR repair pathway after DNA damage.

\section{a}

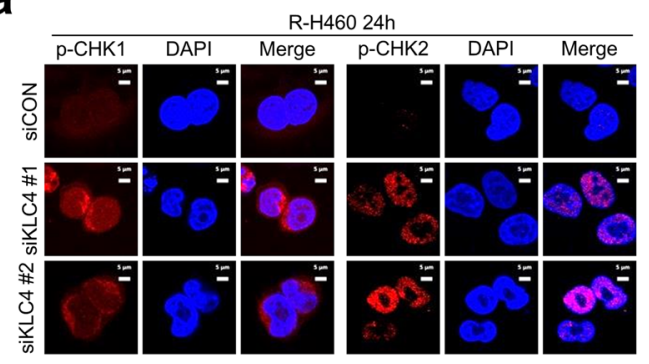

C

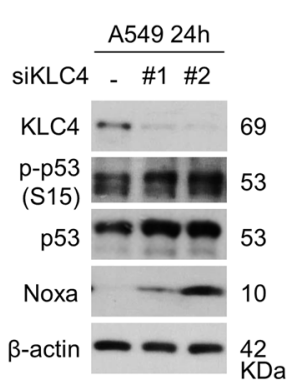

d

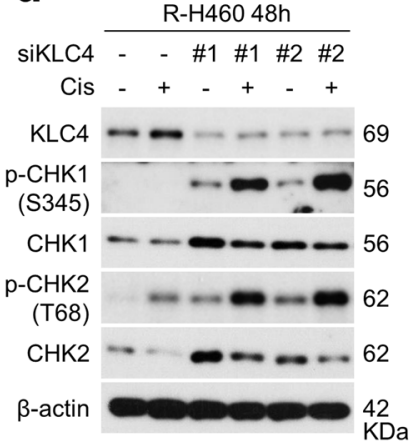

b

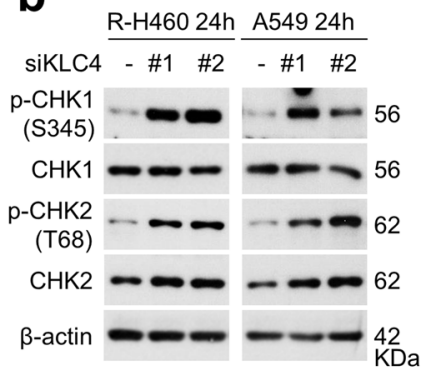

e

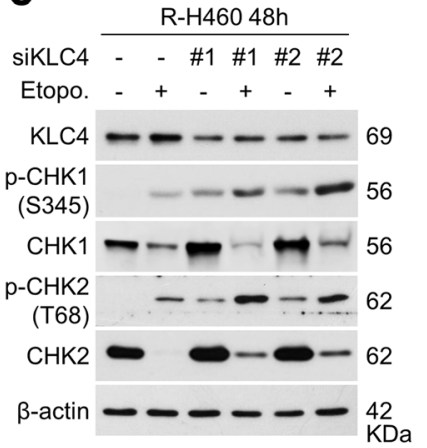

Fig. 4 KLC4 knockdown induced activation of CHK1/CHK2 in R-H460 cells. a Twenty-four hours after transfection with siCON (negative control) or siKLC4, the cells were fixed with paraformaldehyde and immunostained using antibodies targeting p-CHK1 and p-CHK2. b Cell lysates [from cells treated as in (a)] were prepared and used for immunoblotting with antibodies against p-CHK1 (S345), CHK1, p-CHK2 (T68), and CHK2. c Protein levels of KLC4, p-p53 (S15), p53, and Noxa, as determined using western blotting after transfection with KLC4 siRNA. d, e Cells were treated with or without $10 \mu \mathrm{M}$ cisplatin (d) or $10 \mu \mathrm{M}$ etoposide (e) after transfection with KLC4 siRNA. The cell lysates were used for immunoblotting with antibodies against KLC4, p-CHK1 (S345), CHK1, p-CHK2 (T68), and CHK2. 


\section{KLC4 silencing induced $\mathrm{CHK} 1 / 2$ activation}

The above observations suggested that KLC4 may be related to the regulation of the DDR and/or repair of DNA damage. Therefore, we next investigated the effect of KLC4 on activation of CHK1 and CHK2 as the main effectors of DDR signaling ${ }^{11}$. Immunofluorescence observations showed no difference in the pattern of CHK2 and CHK1 phosphorylation after KLC4 siRNA transfection, compared with that after transfection of negative control siRNA in R-H460 cells (Fig. 4a). This indicated that KLC4 may directly affect DDR signaling. Immunoblot analyses demonstrated that the levels of phospho-CHK1 and phospho-CHK2 were higher in KLC4 siRNA-treated cells than in untreated cells (Fig. 4b). As these checkpoint kinases act upstream of $\mathrm{p} 53^{30}$, we studied the phosphorylation status of p53 (Ser15) using western blot analysis; results showed that p53 phosphorylation increased after KLC4 knockdown (Fig. 4c), whereas the total p53 level was not affected. Furthermore, compared with those induced by KLC4 knockdown alone, the combination of KLC4 knockdown and cisplatin/etoposide further increased the levels of phospho-CHK1 and phospho-CHK2 in R-H460 cells (Fig. $4 d$, e). Overall, these results indicated that KLC4 may prevent DNA damage repair and increase DNA damage by inhibiting $\mathrm{CHK} 1 / 2$ in lung cancer cells.

\section{Effect of KLC4 on CHK2-mediated apoptosis}

We next investigated whether KLC4 regulates these functions via $\mathrm{CHK} 2$ as a critical regulator of cell apoptosis and DNA repair ${ }^{31}$. Compared with that observed with siKLC4 treatment alone, the combination of KLC4 knockdown and treatment with a CHK2 inhibitor resulted in a sharp reduction in apoptosis. The protein levels of cleaved PARP and phospho-CHK2 correlated in A549 and R-H460 cells (Fig. 5a, b, d, e). However, no further decrease in apoptosis was observed with depletion of KLC4 and treatment with the CHK1 inhibitor (UCN-01) in A549 and R-H460 cells (Fig. 5c, f). These results showed that KLC4 controls DNA damage-mediated cell apoptosis via $\mathrm{CHK} 2$ and not CHK1.

\section{KLC4 correlated negatively with CHK2 and PRKDC in} samples from patients with lung and colorectal cancer

It was confirmed that the expression of CHK1 and CHK2 was increased when KLC4 siRNA was treated in RH460 cells. Similarly, the mRNA levels of CHK1 and $\mathrm{CHK} 2$ were confirmed to have been affected from the transcription step. By confirming that KLC4 expression inhibition increases the mRNA levels of CHK1 and CHK2, it was found that KLC4 may be involved in the transcriptional step of CHKs (Supplementary Fig. 4a, b). In addition, the correlation between KLC4 and CHEK1, CHEK2 was investigated in both lung cancer and cervical cancer using RNA-sequencing data from publicly available microarray datasets. (Supplementary Fig. 4c and Fig. 6a, d).

Kaplan-Meier analysis showed that high KLC4 and low CHEK2 expression resulted in significantly lower survival rates for patients with lung and colorectal cancer (Fig. 6b, e). There was also a strong negative correlation between the KLC4 mRNA and CHEK2 or PRKDC (related to the NHEJ pathway; DNA-PKcs) mRNA levels in both patient groups (Fig. 6c, f). Together, these findings demonstrated the pivotal roles of KLC4, CHK2, and DNA-PKcs in regulating chemoresistance.

\section{Discussion}

Previously, we had reported that KLC4 depletion induces apoptosis of radioresistant cancer cells via mitochondrial dysfunction due to calcium ion influx. KLC4 knockdown exhibited impaired mitochondrial respiration, increased mitochondrial reactive oxygen species production, and enhanced mitochondrial calcium uptake, which collectively led to mitochondrial dysfunction. For this reason, we proposed that KLC4, a kinesin superfamily member, may represent a novel and effective anticancer target, particularly for patients that exhibit radioresistance. Given that resistance to anticancer drugs, as well as radiation resistance, is a problem observed in many cancer patients, we aimed to determine whether KLC4 affected anticancer resistance in this study. Our results showed that KLC4 regulated chemoresistance to cisplatin in NSCLC cells.

Adjuvant and neoadjuvant chemotherapy remain the main approaches of systemic treatment for many cancers at both early and advanced stages of the disease ${ }^{32}$. These treatments typically involve the use of DNA-damaging agents, as DNA integrity is important for suitable cell function and proliferation ${ }^{33,34}$. Cisplatin is a representative DNA-damaging agent; as an anthracycline antibiotic that intercalates into DNA, it hinders topoisomerase II progression, resulting in cytotoxicity mostly via inhibition of DNA replication and generation of $\mathrm{DSBs}^{33}$. However, resistance (innate and/or acquired) to cisplatin is common in the treatment of many types of cancer ${ }^{35}$. Therefore, it is important to understand the cellular mechanisms related to chemoresistance for identifying novel therapeutic targets that may prevent acquisition of drug resistance.

Based on the standardized nomenclature by the society of kinesin researchers, the human kinesin superfamily has 14 members $^{36}$. However, the nomenclature of kinesin is ambiguous, resulting in several designations and redundant names for a single kinesin. Individual kinesins play an essential role in the intracellular transport of membranous organelles for regulating morphogenesis and ensuring proper function of the cell and protein complexes in a microtubule- and ATP-dependent manner. Furthermore, kinesins have been shown to play vital roles 
a

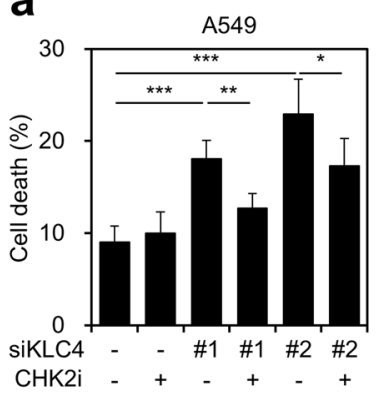

d

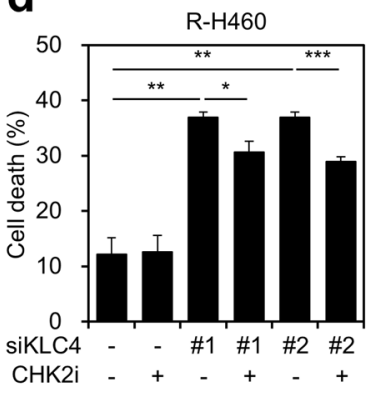

b

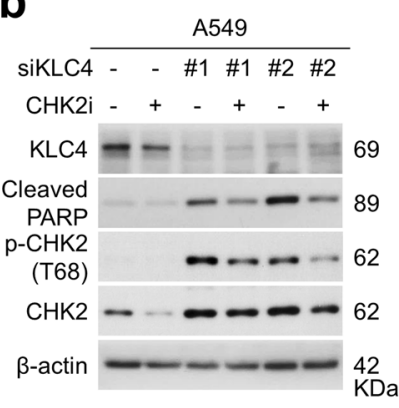

e

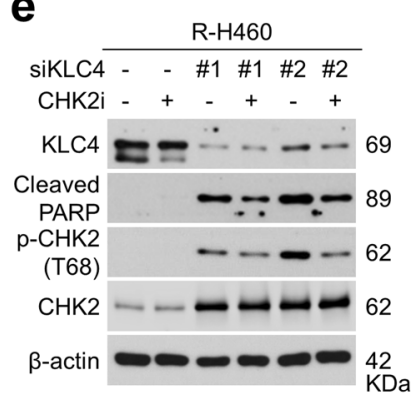

C

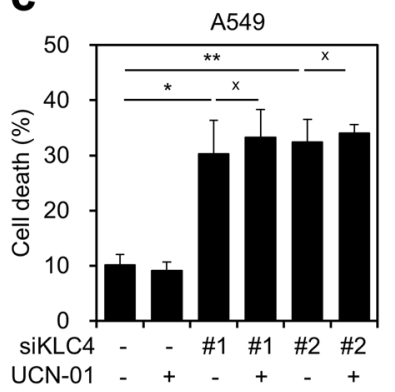

f

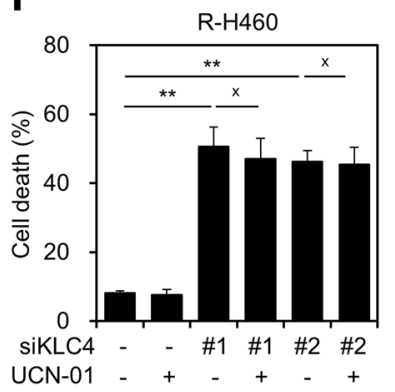

Fig. 5 KLC4 knockdown induced cell death via CHK2 activation. a, b A549 cells were treated with the CHK2 inhibitor (CHK2i), transfected with siCON or siKLC4, and incubated for $48 \mathrm{~h}$. Cell death was determined using annexin V/propidium iodide (AV/PI) staining (a). Levels of the indicated proteins were determined using western blotting (b). c A549 cells were treated with the CHK1 inhibitor (UCN-01), transfected with siCON or siKLC4, and incubated for $48 \mathrm{~h}$. Cell death was determined using AV/PI staining. d R-H460 cells were treated with the CHK2i after transfection with KLC4 siRNA. Cell death in R-H460 cells was determined using AV/PI staining. e Protein levels of the indicated proteins were determined using western blotting after cells were treated as in (d). $\mathbf{f}$ R-H460 cells were treated with the CHK1 inhibitor UCN-01, transfected with KLC4 siRNA, and incubated for $48 \mathrm{~h}$; cell death was determined using AV/PI staining.

in many cellular functions regarding cell division and membrane trafficking events, including endocytosis and transcytosis ${ }^{37-39}$. Although members of the kinesin family vary in shape, a typical kinesin occurs as a protein dimer (molecular pair) consisting of two heavy chains and two light chains ${ }^{40}$. KLC4 belongs to the kinesin light chain family and is a microtubule-associated force-producing protein that has been suggested to play a critical role in organelle transport ${ }^{40,41}$. The light chain may function in coupling of cargo to the heavy chain or in the modulation of its ATPase activity ${ }^{36}$. However, to the best of our knowledge, this is the first study to focus on the biological chemoresistance function of KLC4 in cancer. Functions of KLC4 have not been extensively reported; however, Li et al. recently reported that SETD3 reduces KLC4 expression to improve the sensitization of cervical cancer cells to radiotherapy, and that SETD3 down-regulates $K L C 4$, thus contributing to the radiosensitivity of cervical cancer cells. Hence, it can be inferred that KLC4 overexpression can abolish the regulatory role of SETD ${ }^{42}$. This result, in turn, is consistent with the finding that $K L C 4$ is involved in radioresistance.

Our results showed that up- or downregulation of KLC4 can affect cancer cell proliferation. Expression of KLC4 was also related to the sensitivity of lung cancer cells to chemotherapy. Thus, inhibiting KLC4 may be a new strategy for overcoming chemoresistance in lung cancer cells and simultaneously enhancing the effects of chemotherapy in patients with lung cancer. Furthermore, we demonstrated that these effects of KLC4 are mediated via regulation of $\mathrm{CHK} 2$, which affects the DNA-damaging action of chemotherapeutic drugs. Several studies have shown that CHK2 plays an important role in the maintenance of G2/M arrest and induction of apoptosis in response to radiation therapy or chemotherapy, as lack of CHK2 compromised the G2/M checkpoint and apoptosis, subsequently resulting in chemoresistance $e^{11,31,43-45}$. In agreement with the results of these previous reports, we observed that depletion of $K L C 4$ resulted in CHK $1 / 2$ activation. However, knockdown of KLC4 in cells treated with the CHK1 inhibitor had no further effects on chemoresistance. Thus, we suggested that KLC4 siRNA treatment induced cell death via CHK2, and not CHK1, activation.

Overall, our observations show that the KLC4-CHK2 axis might act as a potential target for developing novel anticancer therapeutics and as a candidate biomarker of tumor chemoresistance. These independent observations 

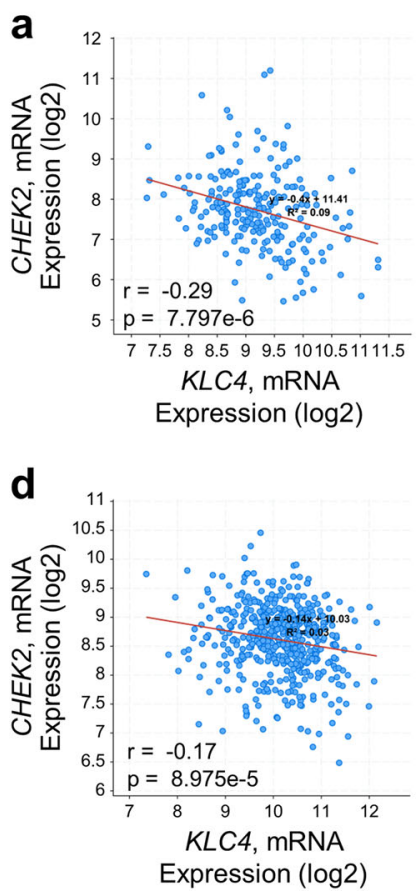
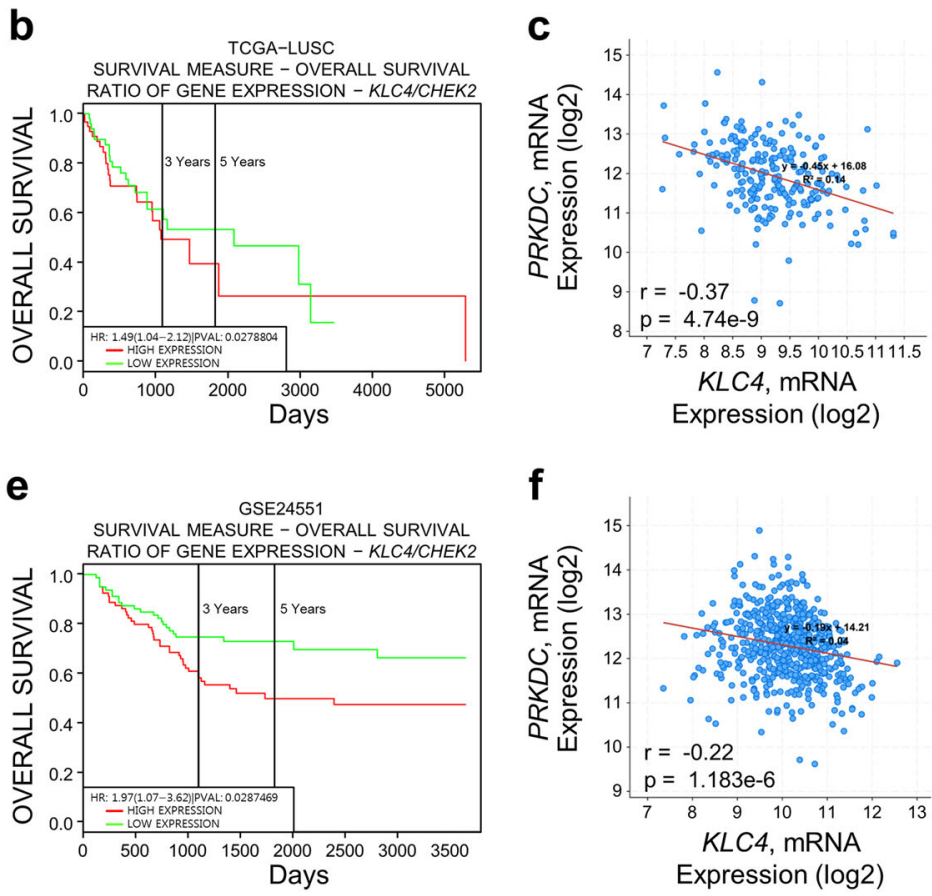

Fig. 6 Correlation of KLC4-CHEK2 signaling with the survival rate of patients with lung cancer. a Correlation of KLC4 with CHEK2 transcripts in the cBioportal datasets of patients with lung cancer (TCGA, Nature 2014). b Effects of KLC4/CHEK2 expression on the overall survival of patients with lung cancer using Kaplan-Meier plotter analysis. c Correlation of KLC4 with PRKDC transcript levels in the cBioportal datasets of patients with lung cancer. d Correlation of KLC4 with CHEK2 transcript levels in the CBioportal datasets of patients with colorectal cancer (TCGA, PanCancer Atlas). e Effects of KLC4/CHEK2 expression on the overall survival of patients with colorectal cancer using Kaplan-Meier plotter analysis. $\mathbf{f}$ Correlation of KLC4 with PRKDC transcript levels in the cBioportal datasets of patients with colorectal cancer.

showed that a comprehensive analysis of the expression levels of chemoresistance-related genes in lung cancer cells may be advantageous in predicting the cellular chemoresistance and cross-resistance to chemotherapeutic agents, which may improve selection of effective drugs for therapy of patients with lung tumors. In this regard, the present study provides new insights into the crosstalk between the CHK2 pathway and the KLC4-regulated apoptotic pathway, which contributes to our understanding of the mechanism underlying the development of drug resistance in cancer cells. The mechanism by which KLC4 can induce the expression of CHK1 and CHK2 is planned to be studied in the future, to determine whether or not mitochondrial dysfunction or other functions affect KLC4. KLC4 is a protein that belongs to the light chain of the motor protein kinesine, which, in turn, is involved in the binding of cargo (protein) carried by the motor protein. This suggests that CHK $1 / 2$ may be involved in the activation of the proteins involved in the activation of CHK1/2. We are conducting further studies to identify the binding protein of KLC4. Furthermore, this study indicates that treatment regimens should be planned according to the specific characteristics and genetic background of each patient, highlighting the need for identifying effective biomarkers for cisplatin resistance.
In conclusion, we propose that chemotherapy might be more effective when combined with RNAi-mediated knockdown of KLC4. For practical applications of this therapeutic strategy, a good vector system has to be developed and tested. In addition, investigations on the effects of KLC4 inhibition on normal cells are required to minimize any potential complications of its inhibition in clinical application for lung cancer treatment.

\section{Materials and methods \\ Cell culture and treatment}

Human lung cancer (A549, H460) cell lines were purchased from the American Type Culture Collection (Manassas, VA, USA). The lung cancer cells were cultured in Roswell Park Memorial Institute (RPMI)-1640 medium supplemented with $10 \%$ fetal bovine serum and $1 \%$ antibiotic-antimycotic. The radioresistant cell line R$\mathrm{H} 460$, derived from parental radiosensitive H460 lung cancer cells cumulatively administered 2-Gy radiation twice a week for 20 weeks, were also used ${ }^{20}$. The direct repeat-green fluorescent protein (DR-GFP) and endjoining (EJ)-GFP-expressing reporter cell lines derived from the U2OS cells transfected with pHRPT-DRGRP and pimEJ5GFP plasmids, respectively, were provided by Dr. Jae-Hoon Ji (Ajou University School of Medicine, 
Suwon, South Korea). The cells were irradiated using a 137Cs source (Atomic Energy of Canada, Ltd., Canada) at a dose rate of $3.81 \mathrm{~Gy} / \mathrm{min}$ and treated with cisplatin, etoposide, gefitinib, and Taxol (Sigma, St. Louis, MO, USA).

\section{Evaluation of apoptosis using annexin V/propidium iodide staining}

In order to quantify cell death caused by experimental treatments as mentioned above, the cells were seeded at a density of $3 \times 10^{5}$ cells per 60 -mm dish and subjected to treatments, as described in experimental conditions. After being incubated in accordance with experimental conditions, trypsin-EDTA was treated with the cells and then washed with phosphate buffered saline. Cells were transferred to $\mathrm{lx}$ binding buffer before being incubated with Annexin V-FITC and PI-PE and analyzed by FACScan flow cytometer (BD Biosciences, San Jose, CA, USA).

\section{Cell viability assay}

The cells were seeded at 5000 cells/well in a 96-well plate, subjected to various treatments as mentioned above, and incubated for $24 \mathrm{~h}$. In order to quantify cell viability, Cyto XTM reagent (LPS solution) was treated for $2 \mathrm{~h}$ and then quantified using Multiscan EX (Thermo) at $450 \mathrm{~nm}$.

\section{Immunofluorescence confocal microscopy}

Immunofluorescence staining for KLC4 (Sigma, St. Louis, MO, USA), $\gamma \mathrm{H} 2 \mathrm{AX} 3$ [a marker of DNA doublestranded breaks (DSBs); Merck KGaA, Darmstadt, Germany], p-CHK1(S345), and p-CHK2(T68) (Cell Signaling Technology Inc., Beverly, MA, USA) was performed as described previously ${ }^{46}$. Cell nuclei were identified by staining with 4,6-diamidino-2-phenylindole.

\section{Western blot analysis}

Western blot analyses were performed as described previously ${ }^{47}$ using primary antibodies against KLC4, $\beta$-actin (Sigma, St. Louis, MO, USA), $\gamma \mathrm{H} 2 \mathrm{AX}$ (Merck KGaA, Darmstadt, Germany), p-p53 (S15), p53, Noxa (Santa Cruz Biotechnology Inc., USA), p-CHK1(S345), CHK1, p-CHK2(T68), CHK2, cleaved poly(ADP-ribose) polymerase (Asp214), and active caspase-3 (Cell Signaling Technology Inc., Beverly, MA, USA).

\section{Knockdown of proteins by siRNA}

As described by the manufacturer (Genolution, Seoul, Korea), the following human-specific siRNAs synthesized were used: siKLC4 \#1, 5'-CCG UUC UAU GGA AAA CAU UUU-3' (sense) and 5'-AAU GUU UUC CAU AGA ACG GUU-3' (antisense); siKLC4 \#2, 5'-CCA GAA UAA GUA UAA GGA AUU-3' (sense) and 5'-UUC CUU AUA CUU AUU CUG GUU-3' (antisense). The cells were transfected with $40 \mathrm{nM}$ siRNA using lipofectamine RNAimax based on each experimental condition.

\section{Colony forming assay}

Cell survival was determined by Colony forming assays. Put succinctly, cells were seeded into triplicate $60-\mathrm{mm}$ tissue culture dishes at densities of $4 \times 10^{2}$ cells / dish. Meanwhile, KLC4 siRNA and cisplatin were treated on the cells. After a period of 14 days, colonies generated from viable cells were stained with trypan blue solution, counting of which was done using a colony counter (Imaging Products, Chantilly, VA).

\section{HR and NHEJ repair assays}

The U2OS DR-GFP and EJ-GFP stable reporter cells were transfected with small interfering RNA (siRNA) targeting KLC4 to knockdown its expression. Humanspecific siRNAs were synthesized as per the manufacturer's instructions (Genolution, Seoul, Korea) and used as described previously ${ }^{21,47}$. At the same time, U2OS (DR-GFP, EJ-GFP) reporter cells were transfected with the pCB-ASce plasmid by Prf. Yoon Sil Lee (Ewha Womens University School of Pharmacy, Seoul, South Korea) using Mirus2020 reagent (Mirus Bio LLC, USA) according to the manufacturer's instructions. The GFPpositive cells were counted using flow cytometry analysis $48 \mathrm{~h}$ post-transfection.

\section{Quantitative reverse transcription-PCR (RT-qPCR) analysis}

As described previously ${ }^{20}$, the Quantitative reverse transcription-PCR (RT-qPCR) was performed using the following primer pairs: CHEK1, 5'-CGGTATAAT AATCGTGAGCG- $3^{\prime}$ (sense) and $5^{\prime}$-TTCCAAGGGTT GAGGTATGT-3' (antisense); CHEK1, 5'-GCGCCTG AAGTTCTTGTTTC-3' (sense) and 5'-GCCTTTGGA TCCACTACCAA-3' (antisense); as well as GAPDH, $5^{\prime}$-CATCTCTGCCCCCTCTGCTGA-3' (sense), and 5'-GGATGACCTTGCCCACAGCCT-3' (antisense).

\section{Data mining using the Kaplan-Meier plotter database and cBioportal database}

Kaplan-Meier survival curves related to KLC4/CHEK2 expression were generated for patients with lung cancer and colorectal cancer. Data were analyzed using the KMplotter (http://genomics.jefferson.edu/proggene/). Coexpression data for KLC4, CHEK1, CHEK2, and PRKDC were extracted from The Cancer Genome Atlas RNAsequencing data in the cBioportal database (https://www. cbioportal.org).

\section{Statistical analyses}

Cell culture experiments were repeated at least thrice. All data are shown as the mean \pm standard deviation. Statistical differences between groups were evaluated 
using Student's $t$ test and $P$ value $<0.05$ was considered significant.

\section{Grants}

This study was supported by a grant from the Korea Institute of Radiological and Medical Sciences, which was funded by the Ministry of Science, ICT, and Future Planning, Republic of Korea [grant number: 50531-2020, 50538-2020].

\section{Acknowledgements}

We are very grateful to the Ajou University and Ewha Womens University for providing us with the experimental materials.

\section{Author details}

${ }^{1}$ Radiation Biology Research Team, Research Center, Dongnam Institute of Radiological and Medical Sciences, Busan 46033, Republic of Korea. ${ }^{2}$ Radiation Oncology Branch, National Cancer Institute, NIH, Bethesda, MD, USA. ${ }^{3}$ Division of Radiation Biomedical Research, Korea Institute of Radiological \& Medical Sciences, Seoul 01812, Korea. ${ }^{4}$ Department of Molecular Cell Biology, Sungkyunkwan University School of Medicine, Suwon 440-746, Korea. ${ }^{5}$ Department of Biochemistry, School of Medicine, Daegu Catholic University, 33, 17-gil, Duryugongwon-ro, Nam-gu, Daegu, Korea

\section{Conflict of interest}

The authors declare that they have no conflict of interest.

\section{Publisher's note}

Springer Nature remains neutral with regard to jurisdictional claims in published maps and institutional affiliations.

Supplementary Information accompanies this paper at (https://doi.org/ 10.1038/s41419-020-2592-z).

Received: 21 November 2019 Revised: 5 May 2020 Accepted: 6 May 2020 Published online: 26 May 2020

\section{References}

1. Siegel, R., Ma, J., Zou, Z. \& Jemal, A. Cancer statistics, 2014. CA Cancer J. Clin. 64 9-29 (2014).

2. Wang, Y. Y. et al. Clinical efficacy of CyberKnife combined with chemotherapy and hyperthermia for advanced non-small cell lung cancer. Mol. Clin. Oncol. 1 527-530 (2013).

3. Brahmer, J. R. Immune checkpoint blockade: the hope for immunotherapy as a treatment of lung cancer? Semin Oncol. 41, 126-132 (2014).

4. Syn, N. L., Teng, M. W. L., Mok, T. S. K. \& Soo, R. A. De-novo and acquired resistance to immune checkpoint targeting. Lancet Oncol. 18, e731-e741 (2017).

5. Wang, S., Zimmermann, S., Parikh, K., Mansfield, A. S. \& Adjei, A. A. Current diagnosis and management of small-cell lung cancer. Mayo Clin. Proc. 94, 1599-1622 (2019).

6. Ohashi, R. et al. Interaction between CD44 and hyaluronate induces chemoresistance in non-small cell lung cancer cell. Cancer Lett. 252, 225-234 (2007).

7. Yang, $\mathrm{H}$. et al. The role of NF-E2-related factor 2 in predicting chemoresistance and prognosis in advanced non-small-cell lung cancer. Clin. Lung Cancer 12, 166-171 (2011).

8. Shanker, M., Willcutts, D., Roth, J. A. \& Ramesh, R. Drug resistance in lung cancer. Lung Cancer (Auckl.) 1, 23-36 (2010).

9. Muhammad, N. \& Guo, Z. Metal-based anticancer chemotherapeutic agents. Curr. Opin. Chem. Biol. 19, 144-153 (2014)

10. Pallis, A. G. \& Syrigos, K. N. Epidermal growth factor receptor tyrosine kinase inhibitors in the treatment of NSCLC. Lung Cancer 80, 120-130 (2013).
11. Bartek, J. \& Lukas, J. Chk1 and Chk2 kinases in checkpoint control and cancer. Cancer Cell 3, 421-429 (2003).

12. Gavande, N. S. et al. DNA repair targeted therapy: the past or future of cancer treatment? Pharm. Ther. 160, 65-83 (2016).

13. Jackson, S. P. \& Bartek, J. The DNA-damage response in human biology and disease. Nature 461, 1071-1078 (2009).

14. Zhou, B. B. \& Elledge, S. J. The DNA damage response: putting checkpoints in perspective. Nature 408, 433-439 (2000).

15. Stracker, T. H., Usui, T. \& Petrini, J. H. Taking the time to make important decisions: the checkpoint effector kinases Chk1 and Chk2 and the DNA damage response. DNA Repair 8, 1047-1054 (2009).

16. Lobrich, M. \& Jeggo, P. A. The impact of a negligent G2/M checkpoint on genomic instability and cancer induction. Nat. Rev. Cancer 7, 861-869 (2007).

17. Antoni, L., Sodha, N., Collins, I. \& Garrett, M. D. CHK2 kinase: cancer susceptibility and cancer therapy - two sides of the same coin? Nat. Rev. Cancer $\mathbf{7}$, 925-936 (2007)

18. Williams, L. H., Choong, D., Johnson, S. A. \& Campbell, I. G. Genetic and epigenetic analysis of CHEK2 in sporadic breast, colon, and ovarian cancers. Clin. Cancer Res 12, 6967-6972 (2006).

19. Matsuoka, S. et al. Reduced expression and impaired kinase activity of a Chk2 mutant identified in human lung cancer. Cancer Res. 61, 5362-5365 (2001).

20. Yun, H. S. et al. Radiotherapy diagnostic biomarkers in radioresistant human H460 lung cancer stem-like cells. Cancer Biol. Ther. 17, 208-218 (2016).

21. Sosa Iglesias, V., Giuranno, L., Dubois, L. J., Theys, J. \& Vooijs, M. Drug resistance in non-small cell lung cancer: a potential for NOTCH targeting? Front Oncol. 8, 267 (2018).

22. Huang, C. Y., Ju, D. T., Chang, C. F., Muralidhar Reddy, P. \& Velmurugan, B. K. A review on the effects of current chemotherapy drugs and natural agents in treating non-small cell lung cancer. Biomedicine 7, 23 (2017).

23. Rajeswaran, A., Trojan, A., Burnand, B. \& Giannelli, M. Efficacy and side effects of cisplatin- and carboplatin-based doublet chemotherapeutic regimens versus non-platinum-based doublet chemotherapeutic regimens as first line treatment of metastatic non-small cell lung carcinoma: a systematic review of randomized controlled trials. Lung Cancer 59, 1-11 (2008).

24. Walles, S. A., Zhou, R. \& Liliemark, E. DNA damage induced by etoposide; a comparison of two different methods for determination of strand breaks in DNA. Cancer Lett. 105, 153-159 (1996).

25. Vancevska, A., Douglass, K. M., Pfeiffer, V., Manley, S. \& Lingner, J. The telomeric DNA damage response occurs in the absence of chromatin decompaction. Genes Dev. 31, 567-577 (2017)

26. Shrivastav, M., De Haro, L. P. \& Nickoloff, J. A. Regulation of DNA double-strand break repair pathway choice. Cell Res 18, 134-147 (2008).

27. Vriend, L. E. et al. Distinct genetic control of homologous recombination repair of Cas9-induced double-strand breaks, nicks and paired nicks. Nucleic Acids Res. 44, 5204-5217 (2016).

28. Ha, G. H. et al. Pellino1 regulates reversible ATM activation via NBS1 ubiquitination at DNA double-strand breaks. Nat. Commun. 10, 1577 (2019).

29. Munoz, M. C., Yanez, D. A. \& Stark, J. M. An RNF168 fragment defective for focal accumulation at DNA damage is proficient for inhibition of homologous recombination in BRCA1 deficient cells. Nucleic Acids Res. 42, 7720-7733 (2014).

30. Mirzayans, R., Andrais, B., Scott, A. \& Murray, D. New insights into p53 signaling and cancer cell response to DNA damage: implications for cancer therapy. J. Biomed. Biotechnol. 2012, 170325 (2012).

31. Zannini, L., Delia, D. \& Buscemi, G. CHK2 kinase in the DNA damage response and beyond. J. Mol. Cell Biol. 6, 442-457 (2014).

32. Yamasaki, M. et al. Multicenter randomized phase II study of cisplatin and fluorouracil plus docetaxel (DCF) compared with cisplatin and fluorouracil plus Adriamycin (ACF) as preoperative chemotherapy for resectable esophageal squamous cell carcinoma (OGSG1003). Ann. Oncol. 28, 116-120 (2017).

33. Cheung-Ong, K., Giaever, G. \& Nislow, C. DNA-damaging agents in cancer chemotherapy: serendipity and chemical biology. Chem. Biol. 20, 648-659 (2013).

34. Pan, Y. et al. Targeting autophagy augments in vitro and in vivo antimyeloma activity of DNA-damaging chemotherapy. Clin. Cancer Res. 17, 3248-3258 (2011).

35. Yoshino, I. Substantial risk affects the stage-dependent outcomes of cisplatinbased adjuvant chemotherapy for completely resected non-small cell lung cancer. Surg. Today 44, 302-306 (2014).

36. Vale, R. D., Reese, T. S. \& Sheetz, M. P. Identification of a novel force-generating protein, kinesin, involved in microtubule-based motility. Cell 42, 39-50 (1985). 
37. DeBoer, S. R. et al. Conventional kinesin holoenzymes are composed of heavy and light chain homodimers. Biochemistry 47, 4535-4543 (2008).

38. Hirokawa, N. Kinesin and dynein superfamily proteins and the mechanism of organelle transport. Science 279, 519-526 (1998).

39. Rahman, A., Kamal, A., Roberts, E. A. \& Goldstein, L. S. Defective kinesin heavy chain behavior in mouse kinesin light chain mutants. J. Cell Biol. 146 1277-1288 (1999).

40. Miki, H., Setou, M., Kaneshiro, K. \& Hirokawa, N. All kinesin superfamily protein, KIF, genes in mouse and human. Proc. Natl Acad. Sci. USA 98, 7004-7011 (2001).

41. Sharp, D. J., Rogers, G. C. \& Scholey, J. M. Microtubule motors in mitosis. Nature 407, 41-47 (2000)

42. Li, Q., Zhang, Y. \& Jiang, Q. SETD3 reduces KLC4 expression to improve the sensitization of cervical cancer cell to radiotherapy. Biochem Biophys. Res Commun. 516, 619-625 (2019).
43. Gogineni, V. R. et al. Chk2-mediated G2/M cell cycle arrest maintains radiation resistance in malignant meningioma cells. Cancer Lett. 313, 64-75 (2011).

44. Hirao, A. et al. DNA damage-induced activation of p53 by the checkpoint kinase Chk2. Science 287, 1824-1827 (2000).

45. Squatrito, M. et al. Loss of ATM/Chk2/p53 pathway components accelerates tumor development and contributes to radiation resistance in gliomas. Cancer Cell 18, 619-629 (2010).

46. Kim, M. J. et al. Depletion of end-binding protein 1 (EB1) promotes apoptosis of human non-small-cell lung cancer cells via reactive oxygen species and Bax-mediated mitochondrial dysfunction. Cancer Lett. 339, 15-24 (2013).

47. Kim, J. S. et al. Chloride intracellular channel 1 identified using proteomic analysis plays an important role in the radiosensitivity of HEp-2 cells via reactive oxygen species production. Proteomics 10, 2589-2604 (2010) 